\title{
Pirlimycin Hydrochloride
}

National Cancer Institute

\section{Source}

National Cancer Institute. Pirlimycin Hydrochloride. NCI Thesaurus. Code C73846.

The hydrochloride salt form of pirlimycin, a derivative of clindamycin with a 6-membered ring replacing clindamycin's 5-membered ring. Compared to clindimycin, pirlimycin has increased activity against gram-positive bacteria, including Staphylococcus aureus and coagulase negative species of Staphylococcus and Streptococcus. This agent is primarily used in the treatment of mastitis in cattle. 